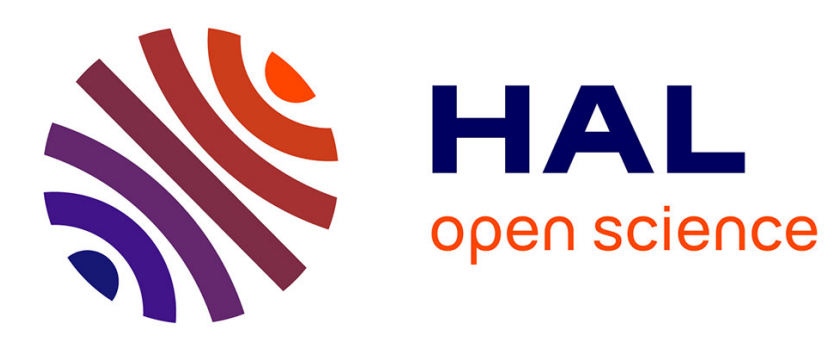

\title{
Mesure de la température de particules en mouvement par pyrométrie photographique monochromatique
}

\author{
D. Hernandez, C. Bonet, A. Charette
}

\section{To cite this version:}

D. Hernandez, C. Bonet, A. Charette. Mesure de la température de particules en mouvement par pyrométrie photographique monochromatique. Revue de Physique Appliquée, 1979, 14 (12), pp.985992. 10.1051/rphysap:019790014012098500 . jpa-00244689

\section{HAL Id: jpa-00244689 https://hal.science/jpa-00244689}

Submitted on 1 Jan 1979

HAL is a multi-disciplinary open access archive for the deposit and dissemination of scientific research documents, whether they are published or not. The documents may come from teaching and research institutions in France or abroad, or from public or private research centers.
L'archive ouverte pluridisciplinaire HAL, est destinée au dépôt et à la diffusion de documents scientifiques de niveau recherche, publiés ou non, émanant des établissements d'enseignement et de recherche français ou étrangers, des laboratoires publics ou privés. 


\title{
Mesure de la température de particules en mouvement par pyrométrie photographique monochromatique
}

\author{
D. Heınandez $(*)$, C. Bonet $(*)$ et A. Charette $\left({ }^{* *}\right)$ \\ (*) Laboratoire des Ultra-Réfractaires, C.N.R.S., B.P. n ${ }^{\circ}$ 5, Odeillo, 66120 Font-Romeu, France \\ $\left({ }^{* *}\right)$ Université du Québec à Chicoutimi, Département des Sciences Appliquées, \\ 930 Est rue J.-Cartier, Chicoutimi, Québec G7H 2B1, Canada
}

(Reçu le 16 juillet, accepté le 24 septembre 1979)

\begin{abstract}
Résumé. - La température de particules fines et chaudes $\left(T>1000^{\circ} \mathrm{C}\right)$ en mouvement lent $(1 \mathrm{~m} / \mathrm{s})$ dans un écoulement, peut être mesurée de façon simple par pyrométrie photographique. Nous avons mis au point une méthode qui consiste en la prise de clichés de la source mobile au travers d'un micropyromètre optique à $\lambda=0,65 \mu \mathrm{m}$. Dans ce cas chaque cliché comprend une référence interne qui est le filament du pyromètre. Nous avons démontré la validité de la méthode en simulant la particule par un diaphragme circulaire $(\varphi=0,2 \mathrm{~mm})$ animé d'un mouvement de translation à vitesse constante et éclairé par une lampe à ruban de tungstène. La précision de la méthode est de l'ordre de $2 \%$ à $1500^{\circ} \mathrm{C}$.
\end{abstract}

\begin{abstract}
The temperature of fine and hot particles $\left(T>1000^{\circ} \mathrm{C}\right)$ moving inside a gas flow at low velocity $\left(1 \mathrm{~ms}^{-1}\right)$ can be measured by photographic pyrometry. We have developed a method which consists in taking pictures of the moving source through an optical micro-pyrometer at $\lambda=0.65 \mu \mathrm{m}$. Each picture contains therefore an internal temperature reference which is the filament of the pyrometer. We have demonstrated the validity of this method by simulation : a circular diaphragm $(\varphi=0.2 \mathrm{~mm})$, translated at constant velocity is illuminated by a tungsten ribbon lamp. Accuracy of the proposed method is $2 \%$ at $1500{ }^{\circ} \mathrm{C}$.
\end{abstract}

I. Introduction. - La mesure par voie optique de la température d'un corps en mouvement pose un délicat problème du fait du mouvement relatif de la source par rapport au récepteur. Le problème se complique lorsque la source, de faible dimension, peut être considérée comme un objet ponctuel. Le flux lumineux reçu dépend alors non seulement de la température de la particule, mais aussi de sa vitesse, de son diamètre et de son facteur d'émission. Lorsque la vitesse de la particule n'est pas très importante $\left(1 \mathrm{~m} . \mathrm{s}^{-1}\right)$ les techniques photographiques paraissent bien appropriées, et nous décrirons dans ce qui suit une méthode photopyrométrique pour la mesure de la température de particules isolées en mouvement.

Dans le cas de vitesses plus importantes, telles que celles couramment rencontrées dans les chalumeaux à plasma (où l'on a mesuré des vitesses axiales pouvant atteindre $300 \mathrm{~m} \cdot \mathrm{s}^{-1}$ pour des particules d'alumine de diamètre 10 à $30 \times 10^{-6} \mathrm{~m}$ ) les méthodes pyrométriques utilisées sont beaucoup plus sophistiquées, compte tenu des conditions physiques mises en jeu. C'est ainsi que (Kruszewska et Lesinski, 1977 [4]) forment l'image d'une zone du jet de plasma sur une fente étroite à l'entrée d'un photomultiplicateur. Les pics d'énergie correspondant au passage isolé des particules sont amplifiés puis envoyés sur un analyseur multicanaux qui donne la distribution des amplitudes des pics. L'étalonnage du système se fait par simulation, à l'aide de trous (d'un diamètre égal à celui des particules), portés par un disque tournant et éclairés par une lampe à ruban de tungstène. La vitesse de rotation du chopper reproduit celle de la particule, de façon à simuler aussi fidèlement que possible le comportement des particules réelles.

(Vardelle, 1979 [7]) a repris et amélioré cette méthode, qu'elle a utilisée (en conjonction avec l'anémométrie Doppler laser pour la mesure de la vitesse axiale et radiale de particules d'alumine injectées dans un chalumeau à plasma) pour la mesure de la température de ces mêmes particules.

Enfin, notons que l'ensemble des méthodes utilisant pour la mesure de la température des particules, le rayonnement émis, donnent accès à la température de luminance (dans le cas de mesures monochromatiques) ou à une température apparente dans le cas général. La détermination de la température réelle 
nécessite, en dernier ressort, la connaissance du facteur d'émission de la particule.

Dans le cas d'oxydes liquides $\left(\mathrm{Al}_{2} \mathrm{O}_{3}, \mathrm{ZrO}_{2}, \mathrm{TiO}_{2} \ldots\right)$ de telles données sont relativement peu nombreuses et se réfèrent, en tout état de cause, aux conditions expérimentales pratiques précisément mises en œuvre lors de la mesure (nature du matériau, composition, nature de l'atmosphère, longueur d'onde...).

II. Principe de la méthode photopyrométrique. La courbe caractéristique d'une émulsion photographique est la représentation graphique de la relation définie par Hurter et Driffield (Gallois D., 1965 [2]) $D=f(\log H)$, avec $D$ : densité optique du négatif impressionné et développé, $H=E \Delta t$ : lumination à laquelle a été soumise l'émulsion, produit de l'éclairement $E$ par le temps d'exposition $\Delta t$. En réalité, la densité optique ne dépend pas du produit $E \Delta t$, mais d'un produit $E \Delta t^{n}$ où $n$ est un exposant voisin de 1 (coefficient de réciprocité de Schwartwild).

Pour un domaine spectral de travail monochromatique, on peut définir les valeurs monochromatiques $D_{\lambda}, H_{\lambda}$ et $E_{\lambda}$. La courbe caractéristique correspondante est illustrée sur la figure 1 où sont indiqués par ailleurs les différents domaines caractérisant le comportement de l'émulsion (sous-exposition, surexposition, solarisation). Dans ces conditions, il existe dans la zone d'exposition correcte - zone $\mathrm{AB}$ - une relation linéaire :

$$
D_{\lambda}=D_{0 \lambda}^{\prime}+\gamma \log E_{\lambda}(\Delta t)^{n}
$$

qui tient compte de l'écart entre les temps de pose $\Delta t$ relatifs aux divers points de la courbe caractéristique.

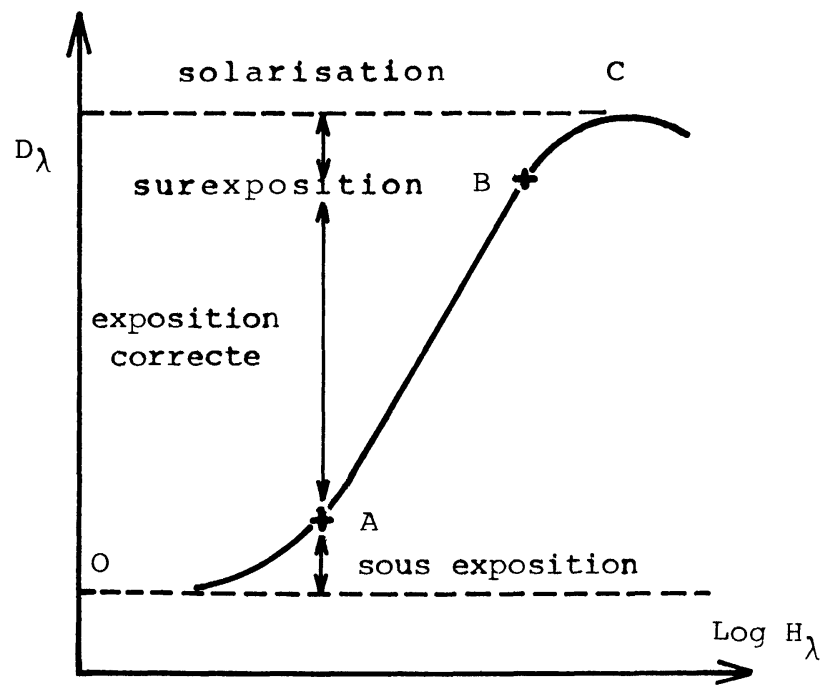

Fig. 1. - Allure générale de la courbe caractéristique d'une émulsion photographique. Variation de la densité optique du négatif $\left(D_{\lambda}\right)$ en fonction du Log de la lumination $\left(H_{\lambda}\right)$.

[General trend for the characteristic curve of a photographic emulsion. Variation of the optical density of the negative $\left(D_{\lambda}\right)$ in terms of the Log of the lumination $\left(H_{\lambda}\right)$.] sion.

La pente du segment $\mathrm{AB}$ est appelée le $\gamma$ de l'émul-

Toutes conditions égales par ailleurs (géométriques, distance, direction, diaphragme, temps de pose, longueur d'onde) $\log E_{\lambda}$ est une fonction linéaire de l'inverse de la température de luminance de la source fixe $T_{l}$. Il vient alors (Villeminot, 1967 [8]) :

$$
D_{\lambda}=D_{0 \lambda}+k \cdot T_{l}^{-1}
$$

$D_{0 \lambda}$ et $k$ sont deux constantes qui dépendent de la longueur d'onde de travail et des caractéristiques de l'émulsion et de plus pour $D_{0 \lambda}$ des conditions de prises de vues.

Pour une source ponctuelle en mouvement rectiligne uniforme, le temps d'exposition $\Delta t^{\prime}$ d'un point $\mathbf{M}$ $\mathrm{du}$ négatif n'est pas fonction du temps de pose $\Delta t$ mais de la vitesse de la particule $v$.

Soient $l$ et $l^{*}$ respectivement la dimension caractéristique de la source dans le sens du déplacement, et la longueur parcourue pendant le temps $\Delta t$; soit $d=l . l^{*-1}$, le facteur de dilution, grandeur directement mesurable sur le cliché.

Il vient :

$$
\Delta t^{\prime}=l . v^{-1}=\left(l . l^{*-1}\right) \cdot \Delta t=d \Delta t .
$$

La densité optique $D$ au point $\mathrm{M}$ s'écrit alors :

$$
D_{\lambda}=D_{0 \lambda}^{\prime}+\gamma \log E_{\lambda}(d \Delta t)^{n} .
$$

Soient $E_{\lambda}^{*}$ et $T^{*}$ respectivement l'éclairement et la température de la source apparents, correspondant à la même densité $D_{\lambda}$ que l'on obtiendrait pour une source immobile, dans des conditions de prises de vues identiques. Il vient par définition :

$$
D_{\lambda}=D_{0 \lambda}^{\prime}+\gamma \log E_{\lambda}^{*}(\Delta t)^{n} \text {. }
$$

En tenant compte de (2), (4) et (5) on trouve (6) :

$$
T_{l}^{-1}=T^{*-1}+n \lambda C_{2}^{-1} \operatorname{Ln} d
$$

avec $C_{2} 2^{\mathrm{e}}$ constante de Planck.

Lorsque le rapport entre $\Delta t$ et $\Delta t^{\prime}$ est inférieur à 10 (soit $d<10$ ) le coefficient de réciprocité de Schwartzwild $n$, peut être pris égal à 1 (Bastie, 1974 [1]). On obtient alors la relation simplifiée (7), qui servira de base à la vérification des résultats expérimentaux.

$$
T_{l}^{-1}=T^{*-1}+\lambda \cdot C_{2}^{-1} \operatorname{Ln} d .
$$

Cette relation montre que l'on peut calculer la température de luminance de la particule en mouvement à partir de la mesure de la température apparente de la particule en mouvement $T^{*}$ et de celle du facteur de dilution $d$. Ces deux mesures sont faites en exploitant directement les clichés. Par ailleurs, la mesure de $T^{*}$ nécessite en général un étalonnage préalable (Villeminot, 1967 [8]). Nous avons mis au point un appareil, le photopyromètre, qui permet de s'affranchir partiellement des causes d'erreur liées à la détermination de $T^{*}$ par photographie. 
III. Le photopyromètre. - Nous avons été amenés, pour appliquer la méthode précédemment décrite, à assembler un " photopyromètre " représenté à la figure 2. Cet appareil résulte de l'association d'un pyromètre à disparition de filament (1) et d'un boîtier d'appareil photographique (2). Il permet donc d'effectuer la prise de vues de la source en mouvement au travers du micropyromètre optique (monochromatique, longueur d'onde effective $\left.0,65 \times 10^{-6} \mathrm{~m}\right)$.

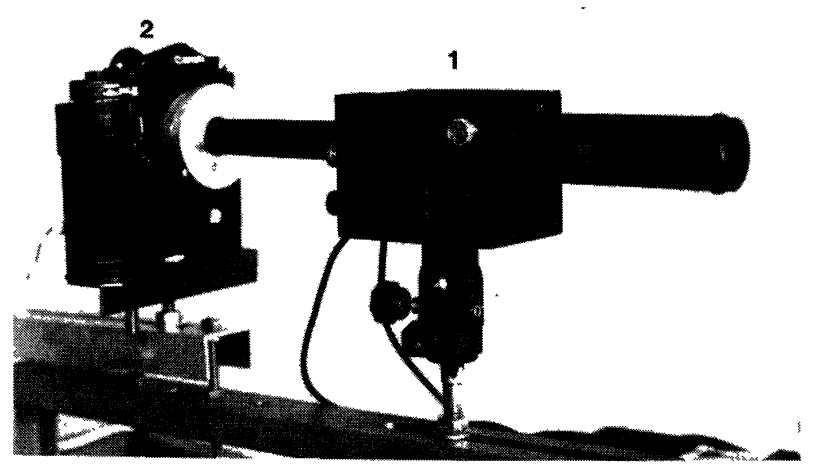

Fig. 2. - Vue du photopyromètre : (1) micropyromètre optique, (2) boîtier d'appareil photographique.

[View of the photo-pyrometer (1) optical micro-pyrometer; (2) camera.]

Le schéma du principe opératoire est sur la figure 3 :

- un système optique permet de superposer dans le plan film les images de la source en mouvement et du filament du pyromètre,

- la température du filament est variable et est contrôlée par la connaissance de la relation couranttempérature,

- le domaine de travail du système est pseudomonochromatique, de longueur d'onde effective $\lambda_{\mathrm{e}}$. Il résulte de la combinaison i) des propriétés de transmission du filtre rouge passe-haut, ii) de la sensibilité spectrale du film utilisé.

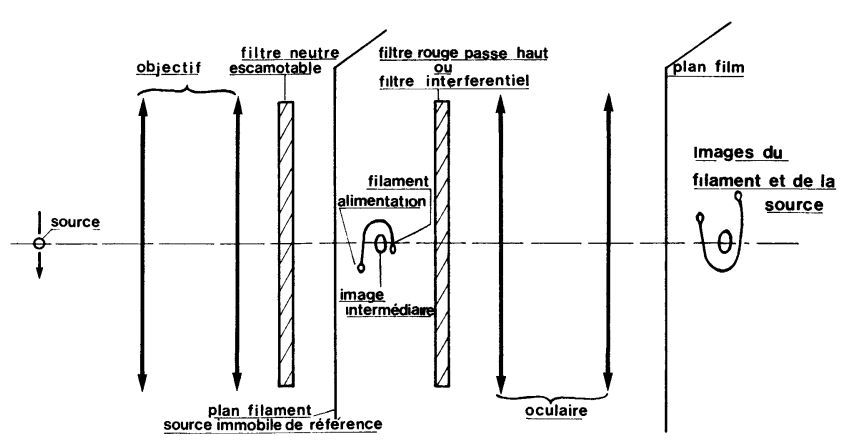

Fig. 3. - Schéma de principe du photopyromètre.

[Scheme for operation of the photo-pyrometer.]
Nous avons utilisé un film commercial panchromatique de sensibilité 400 ASA. Soient $\tau_{\lambda}, V_{\lambda}, S_{\lambda}$ respectivement le facteur de transmission spectrale du filtre rouge, le facteur relatif de visibilité spectrale, le facteur relatif de sensibilité spectrale du film. Ces grandeurs caractérisent directement le comportement spectral du filtre, de l'œil et du film.

On définit pour les combinaisons filtre-œil et filtre-film le facteur d'efficacité spectrale $F_{\lambda}$ :

$$
F_{\lambda}=\frac{\tau_{\lambda} \cdot V_{\lambda}}{\left(\tau_{\lambda} \cdot V_{\lambda}\right)_{\max }} \text { et } F_{\lambda}=\frac{\tau_{\lambda} \cdot S_{\lambda}}{\left(\tau_{\lambda} \cdot S_{\lambda}\right)_{\max }} .
$$

Sur la figure 4 nous avons porté $F_{\lambda}$ en fonction de $\lambda$ pour les deux combinaisons. On voit que les domaines d'efficacité spectrale ainsi définis sont très voisins pour l'œil et le film panchromatique associés au filtre rouge, très différents pour un film infrarouge.

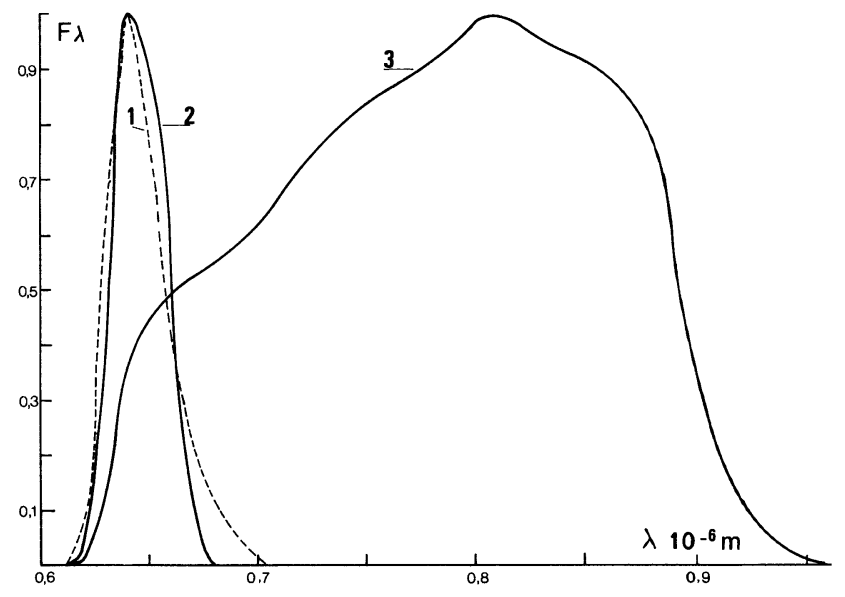

Fig. 4. - Facteur d'efficacité spectrale $F_{\lambda}$ en fonction de la longueur d'onde $(\lambda)$ pour les couples : (1) oil + filtre rouge, (2) film panchromatique + filtre rouge, (3) film infrarouge + filtre rouge.

[Factor for spectral efficiency $F_{\lambda}$ in terms of wavelength $(\lambda)$ for the following combinations : (1) eye + red filter; (2) panchromatic film + red filter; (3) infrared film + red filter.]

Un complément d'information quant au comportement spectral du photopyromètre est fourni par la photographie au travers du photopyromètre de disparitions visuelles obtenues sur source fixe au travers du pyromètre.

Les figures 5 montrent que la disparition visuelle (correspondant à l'égalité des luminances à $0,65 \times 10^{-6} \mathrm{~m}$ pour le filament et la particule) est conservée par le photopyromètre. En effet, la densité optique du filament est égale à celle de la particule. Les mesures sont effectuées par analyse densitométrique des négatifs.

Le domaine pseudomonochromatique de travail $\mathrm{du}$ photopyromètre est caractérisé par la longueur d'onde effective du système. Les mesures précédentes 


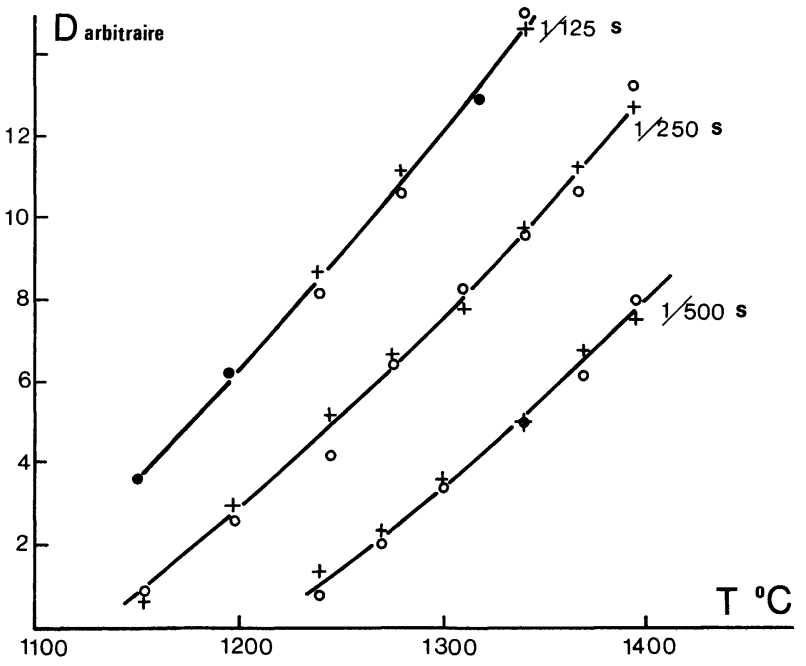

b

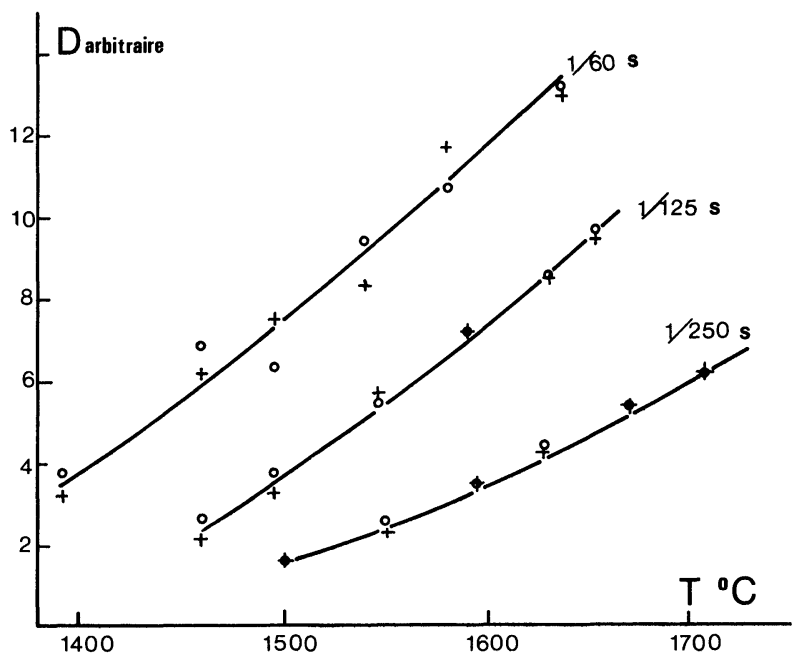

Fig. 5. - Evolution de la densité optique mesurée par densitométrie en fonction de la température de la source fixe mesurée par pyrométrie $(\lambda=0,65 \mu \mathrm{m})$ pour $: a)$ filtre rouge + film panchromatique, $b$ ) filtre rouge + film panchromatique + filtre neutre. O correspond au filament du pyromètre, + à la source fixe. Le temps de pose est indiqué sur chaque courbe.

[Evolution of the optical density measured by densitometry in terms of the temperature of the fixed source measured by pyrometry $(\lambda=0.65 \mu \mathrm{m})$ for : $a)$ red filter + panchromatic film, $b)$ red filter + panchromatic film + neutral filter. $O$ corresponds to the filament of the pyrometer; + to the fixed source. The exposure time is indicated on each respective curve.]

permettent sa détermination en comparant le comportement avec et sans filtre neutre. On trouve :

$$
\lambda_{\mathrm{e}}=0,65 \times 10^{-6} \mathrm{~m} \text {. }
$$

En conséquence, le pyromètre optique et le photopyromètre équipé d'un film panchromatique travaillent à des longueurs d'onde effectives identiques. L'étalonnage du pyromètre (relation courant-température) est donc valable pour le photopyromètre.
En conclusion, l'appareil que nous venons de décrire permet de photographier simultanément une source en mouvement et un filament de référence, de température connue, dans un domaine pseudomonochromatique $\left(\lambda_{\mathrm{e}}=0,65 \times 10^{-6} \mathrm{~m}\right)$.

IV. Le banc de simulation. - Un banc de simulation a été construit pour tester la validité de la méthode décrite. Il assure la translation à vitesse constante, dans le champ du système optique du photopyromètre, d'un diaphragme de diamètre $0,2 \times 10^{-3} \mathrm{~m}$. Ce dernier, éclairé par une lampe à ruban de tungstène, simule le déplacement rectiligne d'une source chaude ponctuelle. L'image de la source mobile est formée par le photopyromètre, dont l'axe optique est en coïncidence avec l'axe diaphragmeruban, grâce à un alignement sur un banc optique.

Le banc de simulation (Fig. 6a) comprend les éléments suivants :

- un banc optique - 1 -,

- une lampe à ruban de tungstène - 2-,

- un dispositif - 3-représenté en détail sur la figure $6 b$ assurant la translation d'un diaphragme (masselotte) - 4-glissant entre deux rails verticaux, dont le mouvement est contrôlé par un contrepoids - 5-, et un électro-aimant $-6-$,

- le photopyromètre - 7-,

- un moteur électrique commandant l'ouverture du photopyromètre $-8-$,

- un système électronique de déclenchement de prises de vues qui permet l'ouverture du diaphragme lors du passage de la source dans le champ du photopyromètre. Ce dispositif comprend une photodiode $-9-$, et un retardateur électronique $-10-$,

- la commande de l'électro-aimant - 11 -.

Le fonctionnement de l'appareil est assuré grâce à la séquence d'opérations suivantes :

- la température $T_{l}$ de la source est fixée par l'intensité traversant le ruban de tungstène,

- la vitesse du diaphragme est ajustée au moyen de la masse du contrepoids,

- le contrepoids choisi est placé en position basse et bloqué par l'électro-aimant. Le diaphragme se trouve en position haute,

- l'électro-aimant libère le contrepoids et le diaphragme descend.

De plus, la synchronisation entre la prise de vues et le passage de la source est réalisée de la façon suivante : lors de la descente au temps $t$ et en un point $M$, la photodiode repère le passage du diaphragme. Le retardateur électronique monté en série entre la photodiode et le moteur transmet le signal électrique avec un retard ajustable $\Delta t$. Le passage de la source dans l'axe du photopyromètre se fait au temps $t+\Delta t$.

Pour une série de prises de vues, il suffit de disposer le contrepoids en position basse après chaque photographie. 
a)
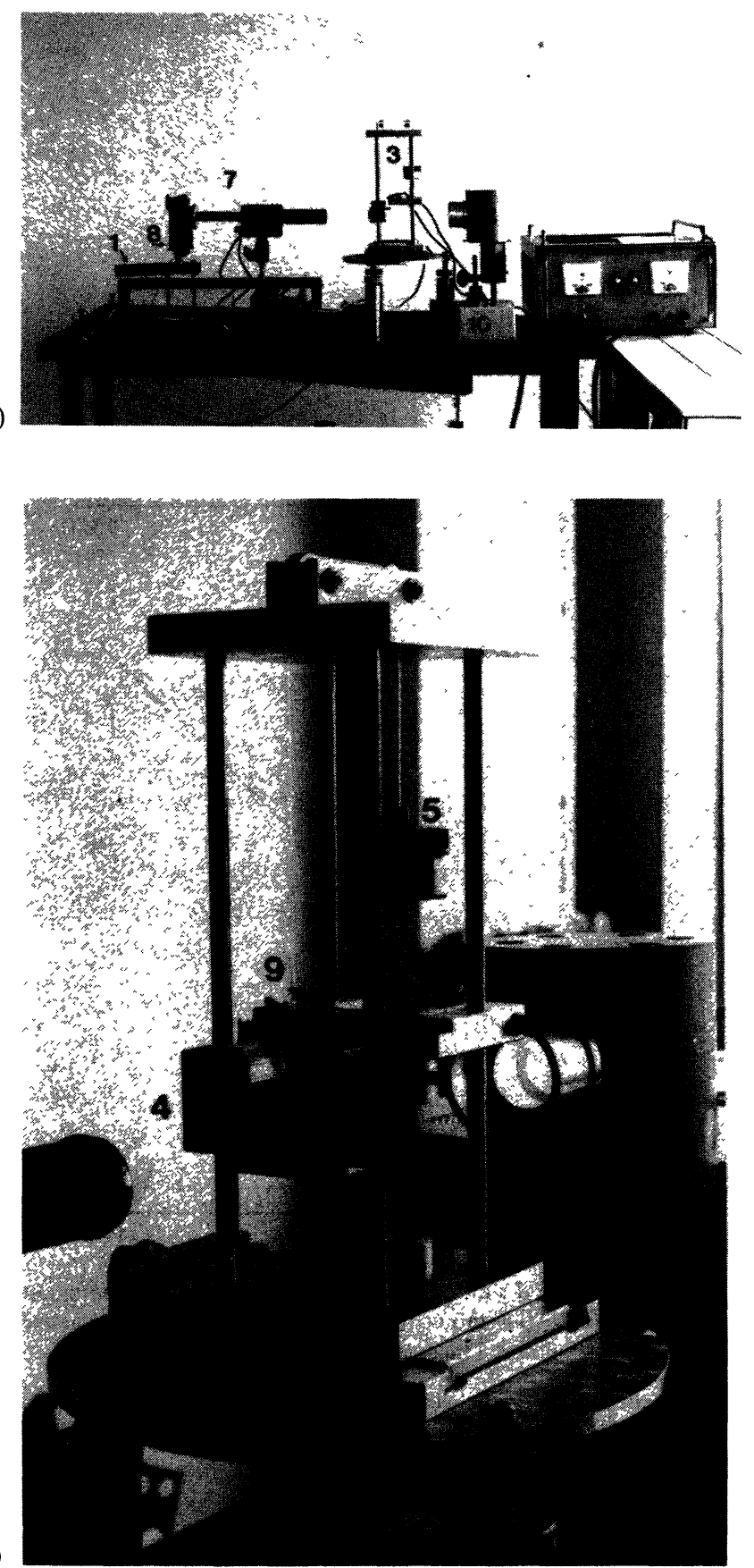

Fig. 6. - - a) Vue du banc de simulation photopyrométrique, b) vue du simulateur de particule. 1 : banc optique; 2 : lampe à ruban de $\mathrm{W} ; 3$ : simulateur de particule ; 4 : porte-diaphragme ; 5 : contrepoids; 6 : électro-aimant $; 7:$ photopyromètre $; 8:$ moteur de commande de prise de vues ; 9 : cellule photoélectrique; $10:$ retardateur électronique; 11 : commande de l'électro-aimant.

[a) View of the photo-pyrometric assembly for simulation, b) view of the apparatus for particle simulation. 1 : optical assembly; 2 : wribbon lamp; 3 : apparatus for particle simulation ; 4 : diaphragm ; 5 : counterweight; 6 : electromagnet; 7 : photo-pyrometer; 8 : motor for driving exposition; 9 : photoelectric cell; 10 : electronic delay; 11 : control for electromagnet.]

V. Résultats expérimentaux. - 1. MeSURES. Nous avons fait trois types de mesure pour démontrer, sur le banc de simulation, la validité de la méthode : i) Mesures pyrométriques classiques sur la source maintenue dans une position fixe. La température de luminance $T_{l}$ ainsi obtenue est une référence à laquelle seront comparés les résultats obtenus à l'aide du photopyromètre.

ii) Mesures pyrométriques avec le photopyromètre, la source étant toujours maintenue en position fixe. La comparaison des résultats obtenus aux mesures pyrométriques (i) après exploitation des clichés, permet de déterminer l'erreur absolue introduite sur la mesure de la température par nos méthodes d'analyse.

iii) Mesures pyrométriques avec le photopyromètre, la source étant alors en mouvement. L'exploitation des clichés permet la détermination de la température apparente de la source en mouvement $T^{*}$ et de la dilution $d$. En reportant dans l'équation (7) on compare les résultats obtenus aux mesures directes (i et ii).

Quant à l'exploitation des clichés, nous l'avons faite de deux façons différentes :

- Etude sur positif : on compare sur des positifs à un grandissement quelconque, le noircissement respectif des images de la source et du filament. On cherche à les rendre identiques en faisant varier la température du filament - disparition sur positif. Lorsque le phénomène de disparition est obtenu, la température du filament correspond à celle de la source. Il s'agit d'une température de luminance $\left(T_{l}\right)$ si la source est immobile, d'une température apparente $\left(T^{*}\right)$ si la source est en mouvement.

La dilution énergétique $d$ est directement mesurée sur le cliché : $d=l . l^{*-1}$. Le principe de cette méthode est illustré sur la figure 7 .

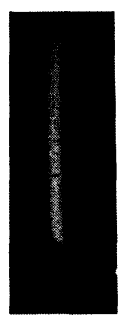

1

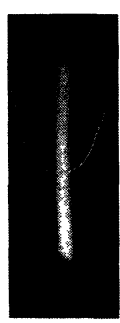

2

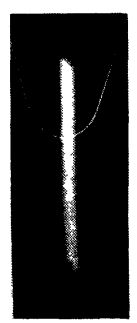

3

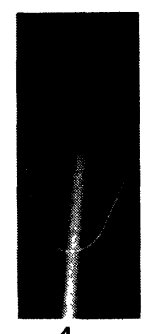

4

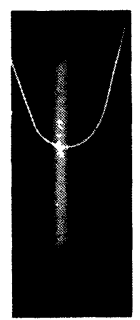

5
Fig. 7. - Clichés montrant le diaphragme en mouvement et le filament de référence. La comparaison du noircissement de la source de température constante à celui du filament de température variable permet de déterminer par disparition la température. Conditions : diamètre du diaphragme $0,2 \mathrm{~mm}$; temps de pose $1 / 250 \mathrm{~s}$; températures du filament : $1: 1275^{\circ} \mathrm{C} ; 2: 1300^{\circ} \mathrm{C}$; $3: 1310^{\circ} \mathrm{C} ; 4: 1325^{\circ} \mathrm{C} ; 5: 1400{ }^{\circ} \mathrm{C}$.

[Views showing the diaphragm in flight and the reference filament. Comparison of the blackening for the source at constant temperature and for the filament at variable temperature allows the determination of the temperature by the so-called disappearing filament technique. Conditions : diameter of the diaphragm $0.2 \mathrm{~mm}$; exposure time $1 / 250 \mathrm{~s}$; temperatures of the filament : $1: 1275^{\circ} \mathrm{C}$; $2: 1300^{\circ} \mathrm{C} ; 3: 1310^{\circ} \mathrm{C} ; 4: 1325^{\circ} \mathrm{C} ; 5: 1400^{\circ} \mathrm{C}$.] 
- Etude sur négatif : on mesure sur le négatif la densité optique du filament pour diverses températures à l'aide d'un densitomètre (type Joyce-Loebl). On en déduit une courbe d'étalonnage $D(T)$, qui correspond à une partie de la courbe caractéristique de l'émulsion. La température de la source est déterminée à partir de cette courbe après mesure de la densité optique de l'image. Les points de la courbe d'étalonnage doivent correspondre à des conditions de prise de vues identiques (temps de pose et diaphragme).

Sur la figure $8 a$ est portée la courbe d'étalonnage pour une source fixe. Sur la figure $8 b$, la courbe d'étalonnage pour une source en mouvement. Ces deux courbes ne sont pas comparables car les conditions de prises de vues sont différentes (temps de pose. et filtre neutre, $1 / 250 \mathrm{~s}$ et $1 / 500 \mathrm{~s}$ ).

Notons que par principe la méthode élimine les causes d'erreur habituelles en photographie dues à la différence de qualité de l'émulsion, de développement des films et de tirage des positifs. En effet, chaque cliché comportant une référence de température interne, ce type d'erreur est, sinon éliminé du moins rendu négligeable.

2. Résultats. - Les résultats des mesures correspondent aux conditions expérimentales suivantes :

- diamètre de la particule simulée par un diaphragme circulaire : $0,2 \times 10^{-3} \mathrm{~m}$,

- vitesse de la particule, $1,3<v<0,5 \mathrm{~m} / \mathrm{s}$,

- film : panchromatique 400 ASA,

- temps de pose : $4 \times 10^{-3} \mathrm{~s}<\Delta t<10^{-3} \mathrm{~s}$.

Dans les tableaux I et II on a rassemblé les résultats suivants :

$T_{l, \mathrm{~m}}$ : température de luminance de la source mesurée directement sur la source fixe,

$T_{l, \mathrm{c}}$ : température de luminance de la source calculée par l'expression (7) à partir des résultats obtenus par photopyrométrie sur la source en mouvement,
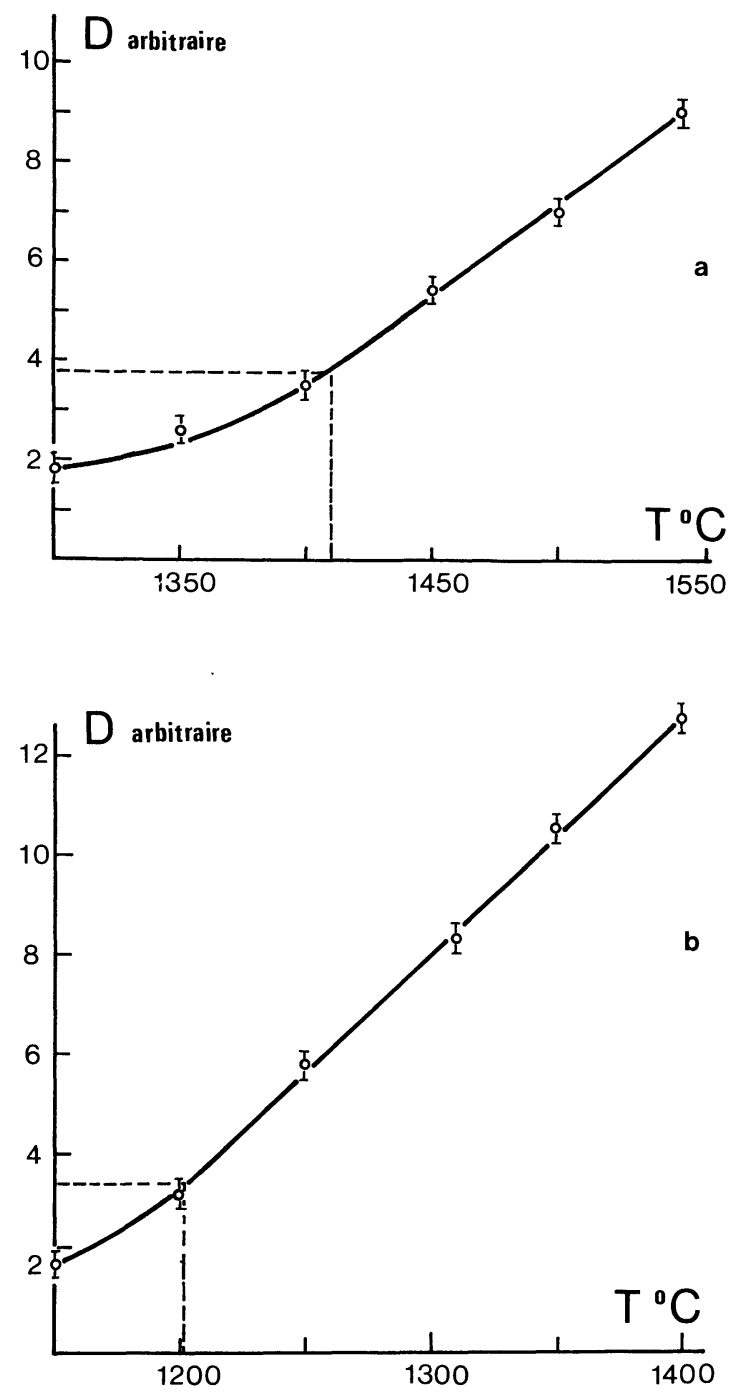

Fig. 8. - Courbe d'étalonnage du film panchromatique pour une source fixe $(a)$ et en mouvement $(b)$, établie par densitométrie. Les segments figurent l'incertitude liée à la mesure.

[Calibration of the panchromatic film for a fixed source $(a)$ and a moving source $(b)$, by optical densitometry. The segments indicate uncertainty upon measurements.]

Tableau I. - Comparaison de la température mesurée directement $\left(T_{l, \mathrm{~m}}\right)$ à celle calculée $\left(T_{l, \mathrm{c}}\right)$ par l'équation (7) $T_{l}^{-1}=T^{*-1}+\lambda^{-1} C_{2} \operatorname{Ln} d$ à partir de la mesure de $T^{*}$ par photopyrométrie sur positif.

\begin{tabular}{lcccccccccc}
\multicolumn{1}{c}{$\begin{array}{c}\text { No du film } \\
-\end{array}$} & 58 & 58 & 50 & 50 & 52 & 56 & 63 & 60 & 10 & 16 \\
$\begin{array}{l}\text { Vitesse de prise de vue : } \Delta T \\
\text { en s }\end{array}$ & $1 / 1000$ & $1 / 500$ & $1 / 250$ & $1 / 500$ & $1 / 250$ & $1 / 1000$ & $1 / 1000$ & $1 / 500$ & $1 / 500$ & $1 / 500$ \\
$\begin{array}{l}\text { Vitesse de la particule } \\
\left(\mathrm{ms}^{-1}\right)\left(^{*}\right)\end{array}$ & 1,24 & 0,96 & 0,62 & 0,60 & 0,61 & 1,20 & 1,28 & 0,82 & 0,57 & 0,56 \\
Facteur de dilution $d\left(^{*}\right)$ & 0,16 & 0,10 & 0,08 & 0,17 & 0,08 & 0,17 & 0,16 & 0,12 & 0,18 & 0,18 \\
$T^{*}$ en ${ }^{\circ} \mathrm{C}$ à $\pm 10^{\circ} \mathrm{C}$ & 1390 & 1340 & 1250 & 1325 & 1305 & 1375 & 1400 & 1395 & 1360 & 1175 \\
$T_{l, \mathrm{c}}$ à $\pm 30^{\circ} \mathrm{C}$ & 1654 & 1658 & 1569 & 1562 & 1647 & 1628 & 1673 & 1709 & 1600 & 1359 \\
$T_{l, \mathrm{~m}}$ à $\pm 10^{\circ} \mathrm{C}$ & 1650 & 1650 & 1600 & 1600 & 1660 & 1660 & 1660 & 1710 & 1590 & 1360 \\
$\Delta\left(T_{l, \mathrm{c}}-T_{l, \mathrm{~m}}\right)$ & +4 & +8 & -31 & -38 & -13 & -32 & +13 & -1 & +10 & -1
\end{tabular}

$\left({ }^{*}\right)$ L'erreur relative sur $v$ et $d$ est d'environ $10 \%$. 
Tableau II. - Comparaison de la température mesurée directement $\left(T_{l, \mathrm{~m}}\right)$ à celle calculée $\left(T_{l, \mathrm{c}}\right)$ par l'équation (7) $T_{l}^{-1}=T^{*-1}+\lambda^{-1} C_{2}$ Lnd à partir de la mesure de $T^{*}$ par photopyrométrie sur négatif.

\begin{tabular}{|c|c|c|c|c|c|c|c|c|c|c|}
\hline No du film & 63 & 63 & 62 & 58 & 58 & 57 & 57 & 56 & 56 & 54 \\
\hline - & - & - & - & - & - & - & - & - & - & - \\
\hline $\begin{array}{l}\text { itesse de prise de vue } \\
\Delta T \text { en s }\end{array}$ & 1000 & $1 / 500$ & $1 / 1000$ & $1 / 1000$ & $1 / 500$ & $1 / 1000$ & $1 / 500$ & $1 / 1000$ & $1 / 500$ & $1 / 500$ \\
\hline $\begin{array}{l}\text { itesse de la particule } \\
v\left(\mathrm{~ms}^{-1}\right)\left({ }^{*}\right)\end{array}$ & 1,25 & 0,98 & 1,12 & 1,25 & 0,96 & 1,36 & 0,92 & 1,17 & 0,9 & 0,70 \\
\hline Facteur de dilution $d\left({ }^{*}\right)$ & 0,16 & 0,10 & 0,18 & 0,16 & 0,10 & 0,15 & 0,11 & 0,17 & 0,11 & 0,14 \\
\hline$T^{*}{ }^{\circ} \mathrm{C}$ à \pm & 1400 & 1340 & 1410 & 1380 & 1340 & 1386 & 1340 & 1405 & 1358 & 1370 \\
\hline$T_{l, \mathrm{~m}}{ }^{\circ} \mathrm{C}$ à $\pm 10^{\circ} \mathrm{C}$ & 1660 & 1660 & 1658 & 1648 & 1648 & 1650 & 1650 & 1650 & 1650 & 645 \\
\hline$T_{l, \mathrm{c}}{ }^{\circ} \mathrm{C}$ à $\pm 30^{\circ} \mathrm{C}$ & 1668 & 1676 & 1664 & 1642 & 1658 & 1664 & 1651 & 1665 & 1673 & 647 \\
\hline$\left(T_{l, \mathrm{c}}-T_{l, \mathrm{~m}}\right)$ & +8 & +16 & +6 & +6 & +10 & +14 & +1 & +15 & +13 & +2 \\
\hline
\end{tabular}

Tableau II (suite).

\begin{tabular}{|c|c|c|c|c|c|c|c|c|c|c|}
\hline No du film & 54 & 49 & 49 & 50 & 50 & 50 & 52 & 52 & 60 & 23 \\
\hline- & - & - & - & - & - & - & - & - & - & \\
\hline Vitesse de prise de vue $: \Delta T$ en $\mathrm{s}$ & $1 / 250$ & $1 / 500$ & $1 / 500$ & $1 / 500$ & $1 / 500$ & $1 / 250$ & $1 / 500$ & $1 / 250$ & $1 / 500$ & $1 / 500$ \\
\hline Vitesse de la particule $v\left(\mathrm{~ms}^{-1}\right)(*)$ & 0,74 & 0,62 & 0,52 & 0,60 & 0,64 & 0,62 & 0,62 & 0,62 & 0,82 & 0,42 \\
\hline Facteur de dilution $d\left({ }^{*}\right)$ & 0,07 & 0,16 & 0,19 & 0,17 & 0,16 & 0,08 & 0,16 & 0,08 & 0,12 & 0,21 \\
\hline$T^{*}{ }^{\circ} \mathrm{C}$ à $\pm 10^{\circ} \mathrm{C}$ & 1270 & 1325 & 1337 & 1346 & 1360 & 1286 & 1383 & 1310 & 1380 & 1222 \\
\hline$T_{l, \mathrm{~m}}{ }^{\circ} \mathrm{C}$ à $\pm 10^{\circ} \mathrm{C}$ & 1645 & 1557 & 1557 & 1600 & 1600 & 1600 & 1640 & 1640 & 1705 & 1410 \\
\hline$T_{l, \mathrm{c}}{ }^{\circ} \mathrm{C}$ à $\pm 30^{\circ} \mathrm{C}$ & 1627 & 1567 & 1556 & 1590 & 1619 & 1621 & 1645 & 1657 & 1695 & 1399 \\
\hline$\Delta\left(T_{l, \mathrm{c}}-T_{l, \mathrm{~m}}\right)$ & -18 & +10 & -1 & -10 & +19 & +21 & +5 & +17 & -10 & 1 \\
\hline
\end{tabular}

(*) L'erreur relative sur $v$ et $d$ est d'environ $10 \%$.

$\Delta\left(T_{l, \mathrm{c}}-T_{l, \mathrm{~m}}\right)$ exprime la différence entre $T_{l, \mathrm{~m}}$ et $T_{l, \mathrm{c}}$ et donne donc un ordre de grandeur de l'erreur commise.

Notons que l'incertitude sur $T_{l, \mathrm{~m}}$ est de $10^{\circ} \mathrm{C}$, tandis que celle sur $T_{l, \mathrm{c}}$ est de $30^{\circ} \mathrm{C}$.

On peut déduire des résultats du tableau I (étude sur positif) et II (étude sur négatif) que, à la précision des résultats près, les résultats expérimentaux sont en accord entre eux. La validité de la méthode étant acquise, il convient maintenant de revenir sur le mode opératoire et de discuter sa précision.

3. Discussion. - Dans ce qui suit nous analysons les caractéristiques principales de la méthode de mesure que nous avons appliquée, en soulignant ses avantages et ses limites :

- Etalonnage du négatif : la prise de vues simultanée de la source en mouvement et du filament de référence permet d'obtenir un étalonnage dans des conditions rigoureusement identiques (géométriques, photographiques et spectrales). De plus, cet étalonnage étant fait pour chaque séquence de prise de vues, nous évitons les délicates manipulations qu'exigent le conditionnement et le traitement des films pour qu'ils conservent leurs propriétés initiales et réagissent dans les mêmes conditions (Urbain et Cabannes 1963 [6]; Shramko, 1967 [5]).
- Détermination de la température apparente $\left(T^{*}\right)$ : nous avons vu que l'on peut exploiter les clichés de deux façons différentes :

a) la méthode sur positif est très souple car le photopyromètre est le seul appareil nécessaire. Le traitement est quasi immédiat avec un système de type Polaroïd. Cependant, l'obtention de la disparition du filament est difficile et nécessite un grand nombre de clichés. Il faut en effet encadrer la température recherchée en utilisant essentiellement des clichés où la trace de la particule coupe le filament.

b) la méthode sur négatif est beaucoup plus régulière car elle nécessite un nombre constant de clichés. En contre-partie, il faut un densitomètre pour analyser les négatifs.

Cette méthode utilise des films commerciaux courants et ne nécessite pas une étude fine de la courbe caractéristique de l'émulsion.

- Imprécision de la méthode : la mise en œuvre nécessite la détermination successive ou simultanée de la température apparente $T^{*}$ et le facteur de dilution $d$.

Des sources d'erreur différentes entachent chacune de ces mesures. Parmi celles-ci on peut citer pour $T^{*}$ : grosseur des grains de l'émulsion, non-reproductibilité du temps d'ouverture du diaphragme, sensibilité de l'analyse densitométrique. L'incertitude globale sur 
la détermination de $T^{*}$ est fournie par la comparaison des températures de sources fixes mesurées par analyse densitométrique et par pyrométrie classique. On trouve $10^{\circ} \mathrm{C}$ autour de $1500^{\circ} \mathrm{C}$.

Quant à la détermination de $d$, les causes d'erreur essentielles sont : défectuosité possible de la mise au point, mauvaise définition de la trace $\left(l^{*}\right)$ du fait de l'ouverture du rideau (phénomène de queue à chaque extrémité de la trace) et non-reproductibilité du temps d'ouverture. Globalement l'incertitude sur la mesure de $d$ peut atteindre $10 \%$, mais sa répercussion sur $T_{l}$ est beaucoup plus faible puisque le terme correspondant intervient sous la forme $\lambda C_{2}^{-1} \operatorname{Ln} d$. Finalement l'incertitude sur $T$ introduite par la mesure de $d$ est du même ordre de grandeur que celle introduite par la mesure de $T^{*}$. Il convient donc d'améliorer simultanément la précision de mesure des deux termes pour améliorer la précision totale de la méthode.

En conclusion, l'erreur relative commise sur la détermination de la température de luminance entre $1350^{\circ} \mathrm{C}$ et $1650^{\circ} \mathrm{C}$ est de l'ordre de $2 \%$.

VI. Conclusion. - Nous avons démontré que le photopyromètre convient à la mesure de la température de luminance d'une source ponctuelle en mouvement. La précision de la méthode est meilleure que $2 \%$ dans la gamme suivante : vitesse $1 \mathrm{~m} . \mathrm{s}^{-1}$, température $1500^{\circ} \mathrm{C}$, diamètre $0,2 \times 10^{-3} \mathrm{~m}$.
Cette méthode ne convient pas pour des vitesses beaucoup plus grandes : en effet, on est alors conduit à diminuer sensiblement le temps de pose, et à utiliser des films rapides. Notons que la longueur d'onde effective du photopyromètre est très différente de celle $\mathrm{du}$ pyromètre si l'on utilise un film infrarouge.

Elle convient par contre, pour des particules plus chaudes sans limitation de température, ou plus froides, mais dans ce cas la limite inférieure est de l'ordre de $1100^{\circ} \mathrm{C}$.

Nous nous sommes placés dans une gamme de conditions opératoires où le rapport de dilution est inférieur à 10. Au-delà de cette limite, le coefficient de réciprocité de Schwartwild doit être pris différent de 1.

Pour l'application à un système réel, il est important de noter que l'on doit tenir compte de la contribution $\mathrm{du}$ milieu propre environnant (lot de particules, gaz rayonnant, surfaces rayonnantes). Le système optique utilisé travaille avec une faible profondeur de champ. Cette propriété, si elle rend difficile la mise au point, offre un avantage pour les mesures locales. La contribution du milieu environnant peut alors être prise en compte comme une correction de fond continu.

Enfin, l'accès à la température réelle de la particule passe inévitablement par la connaissance du facteur d'émission de cette dernière.

Les résultats détaillés de cette étude sont reportés dans la référence (Hernandez, 1978 [3]).

\section{Bibliographie}

[1] BASTIE, J., Radiométrie-Photographie, Techniques de l'Ingénieur, R 3330, 1974.

[2] Gallois, D., Caractéristiques des émulsions photographiques noir et blanc, $2^{\mathrm{e}}$ Colloque National de Cinématographie Rapide, mars 1975.

[3] Hernandez, D., Contribution à la mise au point de méthodes de mesure pour la détermination de la température d'une particule en mouvement et d'un lit fluidisé solaire, Thèse de doctorat de $3^{\mathrm{e}}$ Cycle, Université de Perpignan, 16 décembre 1978.

[4] Kruszewska et Lesinski, Temperature distributions of solid particles in a plasma stream, Revue Phys. Appl. 12 (1977) 1209.
[5] Shranko, Yu. D., Photographic Pyrometry, Teplofizika Vysokikh Temperatur 5 (1967) 367-372.

[6] Urbain, G. et Cabannes, F., Procédés divers de mesures de températures, Techniques de l'Ingénieur R 2650, 1963.

[7] VARdelle, A., Contribution à la mesure statistique des vitesses et des températures de surface de particules injectées dans un jet de plasma d'arc, Thèse de doctorat de $3^{\mathrm{e}}$ Cycle, Université de Limoges, 23 février 1979.

[8] Villeminot, P., Pyrométrie photographique monochromatique, Rev. Int. Htes Temp. et Réfractaires 4 (1967) 5-12. 